\title{
Investigating immunmodulatory mechanisms of cannabinoids: the role of MMP-9
}

\author{
S Tauber*1, R Scheider-Stock ${ }^{2}$ and O Ullrich ${ }^{1}$
}

\begin{abstract}
Address: ${ }^{1}$ University of Zurich, Institut for Anatomy, Department for Cell- and Neurobiology, Zurich, Switzerland and ${ }^{2}$ University of Magdeburg, Institut for Pathology, Department for Molecular Genetics, Magdeburg, Germany

* Corresponding author
\end{abstract}

from I2th Joint Meeting of the Signal Transduction Society (STS). Signal Transduction: Receptors, Mediators and Genes

Weimar, Germany. 29-3I October 2008

Published: 26 February 2009

Cell Communication and Signaling 2009, 7(SuppI I):A89 doi:10.1186/1478-8IIX-7-SI-A89

This abstract is available from: http://www.biosignaling.com/content/7/SI/A89

(C) 2009 Tauber et al; licensee BioMed Central Ltd.

The endocannabinoid system, which comprises endogenous ligands and various receptors, plays an important role in immune control. Endocannabinoid signalling impacts crucial immune cell functions such as migration and chemotaxis and acts as an auto-protective and compensatory system under inflammatory conditions. The inflammatory mediator Matrix-metalloproteinase 9 (MMP-9) helps immune cells to migrate to the sites of inflammation by degrading the extracellular matrix.

The aim of our study was to determine the effect of cannabinoid signalling on the secretion of MMP-9 by macrophages. U937 monocytes were differentiation to macrophages by incubation with PMA (100 nM) for $48 \mathrm{~h}$, and primary human macrophages were isolated from whole blood. Treatment with the synthetic cannabinoid WIN 55, 212-2 (2 or $4 \mathrm{uM}$ ) induced a dose dependent inhibition of MMP-9 secretion as shown by activity assay and western blot analysis of conditioned media. Kinetic analysis of cell lysates by western blot showed intracellular accumulation of MMP-9 going along with the inhibited secretion, proposing inhibition of the secretion process as a mechanism. Quantitative real-time-PCR was used to measure MMP-9 mRNA and revealed a possible negative feedback loop on the transcriptional level which involves MAP-kinases as shown by phospho-specific western blot analysis. The effect could not be blocked by pharmacological inhibition of the known cannabinoid receptors $\mathrm{CB} 1, \mathrm{CB} 2$, and TRPV1, but was stereospecific, as it could not be reproduced with the enantiomer WIN55,
212-3. The effect therefore seems to be mediated by a yet unidentified cannabinoid-binding site.

Our results suggest that cannabinoid-induced inhibition of MMP-9 secretion presents a new mechanism of antiinflammatory action of the cannabinoid system and helps to provide a basis for the development of cannabinoidbased drugs for inflammatory diseases. 